\title{
DNA methyltransferase 3-like affects promoter methylation of thymine DNA glycosylase independently of DNMT1 and DNMT3B in cancer cells
}

\author{
HEESUN KIM ${ }^{1,2}$, JINAH PARK ${ }^{1}$, YEONJOO JUNG ${ }^{1}$, SANG-HYUN SONG $^{1}$, SAE-WON HAN $^{1,3}$, \\ DO-YOUN OH ${ }^{1,3}$, SEOCK-AH IM ${ }^{1,3}$, YUNG-JUE BANG ${ }^{1,3,4}$ and TAE-YOU KIM ${ }^{1,3,4}$ \\ ${ }^{1}$ Cancer Research Institute, Seoul National University College of Medicine; Departments of ${ }^{2}$ Biomedical Sciences, \\ ${ }^{3}$ Internal Medicine, Seoul National University College of Medicine; ${ }^{4}$ Department of Molecular Medicine and \\ Biopharmaceutical Sciences, Graduate School of Convergence Science and Technology \\ and College of Medicine, Seoul National University, Seoul, Korea
}

Received December 30, 2009; Accepted February 26, 2010

DOI: 10.3892/ijo_00000643

\begin{abstract}
DNA methyltransferase (DNMT) 1 and 3 are primarily responsible for abnormal methylation in cancer. Unlike these DNMTs, DNA methyltransferase 3-like (DNMT3L) harbors no conserved catalytic domain, and has been shown to function as a regulatory cofactor for DNA methylation. However, it is unclear whether DNMT3L directly regulates DNA methylation in cancer cells. To address this, we investigated the methylation targets of DNMT3L by conducting methylation microarray trials after the siRNAinduced knockdown. We determined that methylation of 242 out of 1,505 CpG sites was significantly altered by DNMT3L knockdown. Among these $242 \mathrm{CpG}$ sites, 204, 12, and 11 $\mathrm{CpG}$ sites were identified as common targets of DNMT $1 / 3 \mathrm{~B} / 3 \mathrm{~L}, 1 / 3 \mathrm{~L}$, and $3 \mathrm{~B} / 3 \mathrm{~L}$, respectively; this indicates that DNMT3L participates in DNA methylation via cooperation with other DNMTs. However, we also determined that the methylation of $15 \mathrm{CpG}$ sites was significantly altered by DNMT3L knockdown only. As a validation, we confirmed that thymine DNA glycosylase (TDG), an enzyme involved in the base excision repair of mismatched-DNA, was upregulated in DNMT3L knockdown cells, but neither in DNMT1 nor 3B knockdown cells. Methylation-specific PCR (MSP) also showed that promoter methylation of TDG was decreased in DNMT3L knockdown cells. Interestingly, 5aza-2'-deoxycitidine (5-aza-dC) re-expressed DNMT3L, leading to down-regulation of TDG. This study is the first to show that DNMT3L exerts a major effect on the
\end{abstract}

Correspondence to: Dr Tae-You Kim, Department of Internal Medicine, Seoul National University Hospital, 101 Daehang-ro, Jongno-gu, Seoul 110-744, Korea

E-mail: kimty@snu.ac.kr

Key words: DNA methyltransferase 3-like, thymine DNA glycosylase, methylation microarray, DNA methylation, cancer transcriptional regulation of a specific target gene, such as TDG, despite the absence of enzymatic activity.

\section{Introduction}

The aberrant methylation of $\mathrm{CpG}$ islands has been a wellestablished event in the tumorigenic process $(1,2)$. The hypermethylation of promoter $\mathrm{CpG}$ islands in tumor suppressor genes results in transcriptional silencing of these genes (2). By way of contrast, global hypomethylation in repetitive regions contributes to the genomic instability characteristic of tumors (2). These aberrant methylation events in tumorigenesis are mediated by DNMTs including DNMT1, DNMT3A, DNMT3B, and DNMT3L. DNMT1 functions principally in the maintenance of methylation (3), whereas DNMT3A and DNMT3B are thought to carry out de novo methylation (4). In a previous study, the disruption of both DNMT1 and DNMT3B reduced the methylation of genomic DNA by $\sim 95 \%$ (5), thus demonstrating that these two enzymes cooperatively maintained DNA methylation and gene silencing in human cancer cells.

Unlike other DNMTs, DNMT3L harbors no functional catalytic site and has been confidently identified as a regulatory factor of DNMT3A and DNMT3B (6,7). Although DNMT3L lacks enzymatic activity, loss of DNMT3L or DNMT3L mutation results in failures of embryonic development, as well as the establishment of paternal imprints $(8,9)$. This means that DNMT3L may have a critical function on DNA methylation, above and apart from its ancillary function. Some recent studies have demonstrated that the loss of methylation at the DNMT3L promoter occurs in cervical carcinogenesis (10) and that the overexpression of DNMT3L in human cancer cell lines can result in nuclear reprogramming and substantial changes in genome-wide gene expression (11). DNMT3L, as well as other members of the DNMT3 family, are expressed abundantly in embryonic stem (ES) cells, but DNMT3L expression levels are down-regulated in accordance with increases in promoter methylation occurring during embryonic development (12). Thus, the abrupt re-expression of DNMT3L in some usually silenced cancer cell lines suggests that the 
Table I. Primer sequences for RT-PCR and real-time RT-PCR analysis.

\begin{tabular}{|c|c|c|c|}
\hline & Primer sequence $\left(5^{\prime} \rightarrow 3^{\prime}\right)$ & Length (bp) & $\operatorname{AT}\left({ }^{\circ} \mathrm{C}\right)$ \\
\hline $\begin{array}{l}\text { DNMT3A1/3 } \\
\text { (RT-PCR) }\end{array}$ & $\begin{array}{l}\text { F: ACTTGGAGAAGCGGAGTGAG } \\
\text { R: CCTCCATTTTCATGGATTCG }\end{array}$ & 390 & 58 \\
\hline DNMT3A2 & $\begin{array}{l}\text { F: GCTACTTCCAGAGCTTCAGG } \\
\text { R: CGTCTTTCAGGCTACGATCC }\end{array}$ & 130 & 58 \\
\hline $\begin{array}{l}\text { DNMT3A } \\
\text { (real-time) }\end{array}$ & $\begin{array}{l}\text { F: CAGCGTCACACAGAAGCATATCC } \\
\text { R: GGTCCTCACTTTGCTGAACTTGG }\end{array}$ & 433 & 60 \\
\hline DNMT3L & $\begin{array}{l}\text { F: TGAGCAACTGGGTGTGCTAC } \\
\text { R: GGGTCAGAACCACTTTCCAA }\end{array}$ & 235 & 58 \\
\hline NEFL & $\begin{array}{l}\text { F: ATGAATGAAGCGCTGGAGAA } \\
\text { R: TGCCATTTCACTCTTTGTGG }\end{array}$ & 126 & 62 \\
\hline IPF1 & $\begin{array}{l}\text { F: AAGCTCACGCGTGGAAAG } \\
\text { R: GCCGTGAGATGTACTTGTTGAA }\end{array}$ & 144 & 62 \\
\hline BCL2A & $\begin{array}{l}\text { F: GCTGGGAAAATGGCTTTGTA } \\
\text { R: GCTTCAGGAGAGATAGCATTTCA }\end{array}$ & 102 & 60 \\
\hline CDKN1A & $\begin{array}{l}\text { F: CGAAGTCAGTTCCTTGTGGAG } \\
\text { R: CATGGGTTCTGACGGACAT }\end{array}$ & 111 & 60 \\
\hline TDG & $\begin{array}{l}\text { F: GAACCTTGTGGCTTCTCTTCA } \\
\text { R: GTCATCCACTGCCCATTAGG }\end{array}$ & 95 & 58 \\
\hline$B$-actin & $\begin{array}{l}\text { F: TCATCACCATTGGCAATGAG } \\
\text { R: CACTGTGTTGGCGTACAGGT }\end{array}$ & 155 & 60 \\
\hline GAPDH & $\begin{array}{l}\text { F: GGAAGCTTGTCATCAATGGAA } \\
\text { R: TGGACTCCACGACGTACTCA }\end{array}$ & 102 & 60 \\
\hline
\end{tabular}

role of DNMT3L may be as functionally relevant to tumor development as it is to embryonic development. The contributions of DNMT3L and its specific target genes to cancer, however, have yet to be clearly elucidated.

In this study, we found that methylation of 15 out of 1,505 $\mathrm{CpG}$ sites was altered significantly by knockdown of only DNMT3L. Our results showed that, while DNMT3L exerts a minor effect on most genes as cofactors of other DNMT3 family members (DNMT3A and DNMT3B), certain genes, including TDG, were affected primarily by DNMT3L.

\section{Materials and methods}

Cell lines and reagents. The human gastric cancer cell lines SNU5, SNU16, SNU484, SNU601, SNU620 and MKN28 were obtained from the Korean Cell Line Bank. Cells were treated with 5-aza-dC via the addition of a fresh medium containing 5-aza-dC (0.1-5 $\mu \mathrm{M}$; Sigma) every $48 \mathrm{~h}$ for 4 days and RNAs were prepared for further assay. siRNAs specific to each DNMT and nonspecific control (Qiagen) were used at concentrations of $20 \mathrm{nM}$. Transfections were repeated every 2 days for 6 days and conducted using Lipofectamine 2000 (Invitrogen) in accordance with the manufacturer's instructions.
Cell cycle analysis. Cells treated with siRNAs for each DNMT $(20 \mathrm{nM})$ every $48 \mathrm{~h}$ for 6 days were harvested and then fixed with cold $70 \%$ ethanol. Each sample was washed with phosphate-buffered saline (PBS) and stained with $20 \mu \mathrm{g} / \mathrm{ml}$ propidium iodide (PI, Sigma) containing $10 \mu \mathrm{g} / \mathrm{ml}$ RNase A (Sigma). After incubation in the dark for at least $15 \mathrm{~min}$, DNA contents of each sample were analyzed using Flow cytometric analysis (FACSCalibur flow cytometer; BD Biosciences, San Jose, CA, USA). The sub-G1 population of the DNA content was considered as apoptotic cells.

Methylation microarray. DNAs $(1 \mu \mathrm{g})$ obtained from cells treated with each DNMT siRNA every 48 h for 6 days was treated with sodium bisulphate using EpiTect ${ }^{\circledR}$ Bisulfite (Qiagen). The bisulphite-treated DNA was subsequently applied to an Illumina bead array (13) using Illumina Goldengate Methylation Cancer Panel I (Macrogen).

$R T-P C R$ and real-time $R T-P C R$. Total RNA was extracted using the TRI reagent ${ }^{\circledR}$ (Molecular Research Center) in accordance with the manufacturer's instructions. cDNA was synthesized using ImProm-II ${ }^{\mathrm{TM}}$ reverse transcriptase (Promega) and amplified using AmpliTaq ${ }^{\circledR}$ Gold DNA polymerase (Applied Biosystems) with gene-specific primers 
A

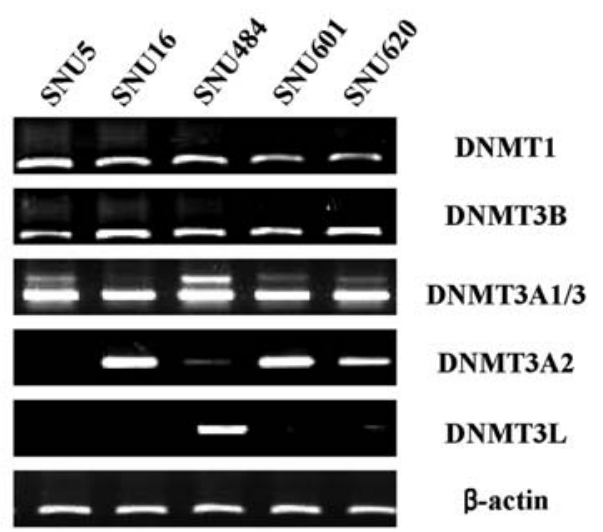

B

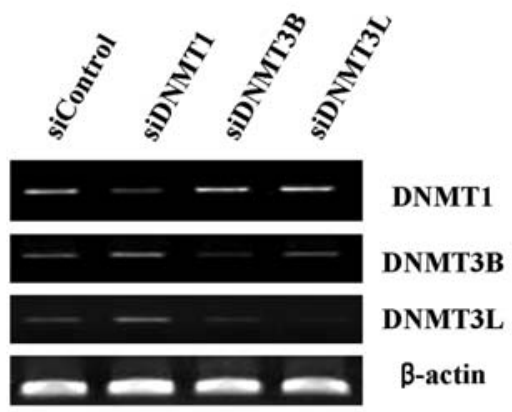

$\mathrm{C}$
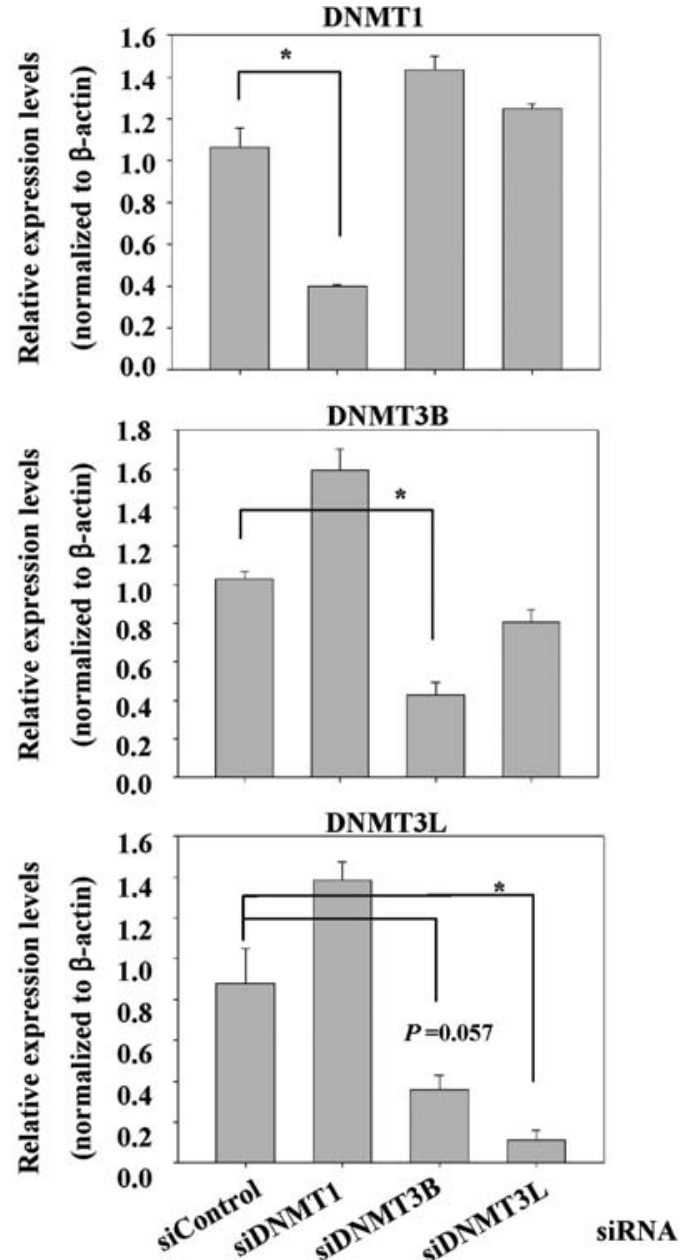

Figure 1. DNMT3L expression in SNU484 gastric cancer cell lines. (A) Basal expression levels of each of the DNMTs in gastric cancer cell lines. RT-PCR was conducted using specific primers for each of the DNMTs. B-actin was used as a loading control. (B and C) Knockdown of each DNMT in SNU484 cells by siRNAs. SNU484 cells were transfected with siRNAs every $48 \mathrm{~h}$ for 6 days. DNMTs mRNA expression levels were confirmed via RT-PCR and real-time RT-PCR and normalized to $ß$-actin. The bars represent the means \pm SD and the control sample is set to a value of $1.0 ;{ }^{*} \mathrm{P}<0.05$.

(Table I). The primers for DNMT1 and DNMT3B were derived as reported previously (14). $\beta$-actin and GAPDH expressions were used as internal controls for the RT-PCR. The levels of each gene mRNA were quantitatively analyzed via real-time RT-PCR assays with SYBR Green I (Molecular Probe) using an iCycler instrument (Bio-Rad) with more than duplet reactions. Each relative mRNA expression level was calculated by normalization to the mean value of $B$-actin.

Methylation-specific PCR. Genomic DNA samples were isolated using a QIAamp ${ }^{\circledR}$ DNA mini kit (Qiagen). Genomic DNA of $1 \mu \mathrm{g}$ was treated with sodium bisulfite with EpiTect Bisulfite (Qiagen). MSP (15) was conducted using AmpliTaq Gold with primers specific to methylated or unmethylated sequences of the genes (Table II).

\section{Results}

Knockdown of DNMT. Although DNMT3L harbors no conserved catalytic domain, some groups have reported that specific $\mathrm{CpG}$ sites were demethylated upon the deletion of DNMT3L (16), thus suggesting that DNMT3L may be involved in the transcriptional regulation of some genes by
Table II. Primer sequences for MS-PCR analysis.

\begin{tabular}{ll}
\hline Gene & \multicolumn{1}{c}{ Primer sequence $\left(5^{\prime} \rightarrow 3^{\prime}\right)$} \\
\hline $\begin{array}{ll}\text { TDG } \\
\text { Methylated }\end{array}$ & $\begin{array}{l}\text { F: GTATTACGCGGTATTATAGAGATC } \\
\text { R: AACCGCTAATACGAACGACTC }\end{array}$ \\
Unmethylated & F: GTATTATGTGGTATTATAGAGATTG \\
& R: AACCACTAATACAAACAACTC \\
\hline
\end{tabular}

altering the levels of DNA methylation. In this regard, we attempted to identify target genes whose methylation levels were altered by DNMT3L. To this end, we utilized SNU484 gastric cancer cells that express DNMT3L mRNA (Fig. 1A).

The expression of DNMTs was blocked via repeated transient transfection of siRNA against each DNMT at $48 \mathrm{~h}$ intervals for a total of 6 days. As shown in Fig. 1B and C, we determined that the expression of each DNMT was downregulated after each siRNA transfection via RT-PCR and quantitative real-time RT-PCR. However, we found that the 


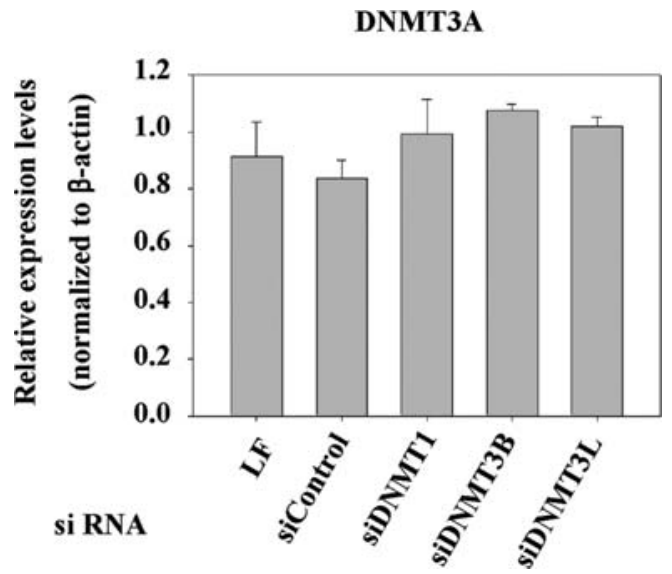

Figure 2. mRNA expression levels of DNMT3A in each DNMT-knockdown cell. Real-time RT-PCR was performed using specific primers for DNMT3A.

knockdown of DNMT3L tended to induce the downregulation of DNMT3B (Fig. 1C). Since the levels of DNMT3A mRNA were almost unchanged after the knockdown of other DNMTs (Fig. 2), we were able to rule out the effects of DNMT3A in our further attempts to identify the target genes regulated by DNMT3L. Interestingly, the apoptosis rate in the SNU484 cells treated with DNMT3L siRNA was as high as in the cells treated with siDNMT1 (Fig. 3). This result indicates that DNMT3L may perform a crucial role in cancer proliferation.

Screening of target genes regulated by DNMT3L. Next, in order to identify target genes specifically regulated by DNMT3L, we conducted GoldenGate methylation microarrays using SNU484 cells. The GoldenGate Assay for methylation allows for the measurement of the DNA methylation status of up to 1,505 targeted $\mathrm{CpG}$ sites from $\sim 800$ genes. Methylation microarray analyses were performed on the basis of the B-value (Fig. 4A). The $\beta$-value refers to methylation levels in

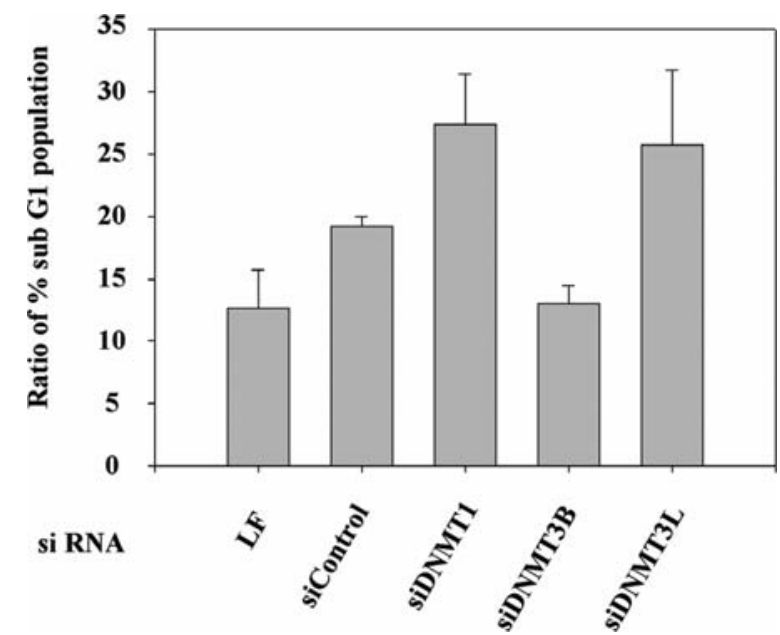

Figure 3. Apoptosis rate of DNMT3L knockdown cells. SNU484 cells were transfected with control, DNMT1, DNMT3B or DNMT3L siRNA every $48 \mathrm{~h}$ for 6 days and measured the ratio of $\%$ subG1 population through FACSCalibur flow cytometer. LF, lipofectamine.

the $\mathrm{CpG}$ sites, ranging from 0 in the case of completely unmethylated sites to 1 in completely methylated sites (13). A numerical difference $>0.17\left(\mid B-\beta_{\text {control }} I \geq 0.17\right)$ refers to a significant difference in the methylation level, as compared to controls. In DNMT3B and DNMT3L knockdown cells, significant changes in methylation levels were detected in $234 \mathrm{CpG}$ sites and 242CpG sites, respectively (Fig. 4B). Interestingly, in the following cases, the majority of these $\mathrm{CpG}$ sites overlapped. The $216 \mathrm{CpG}$ sites were overlapped in the knockdown of DNMT1 and DNMT3L and $217 \mathrm{CpG}$ sites in the knockdown of DNMT3L and DNMT3B. Additionally, among these sites, $204 \mathrm{CpG}$ sites were significantly altered in all of the cells treated with siDNMT1, siDNMT3B, or siDNMT3L (Fig. 4B). These results are bolstered by the fact that each of the DNMTs cooperatively plays a role in DNA methylation and gene silencing $(5,12)$.

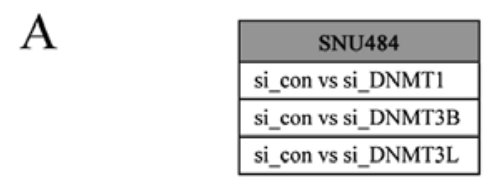

In $1,505 \mathrm{CpG}$ sites

Background Normalization Except for P-value $>0.05 \mathrm{CpG}$ site

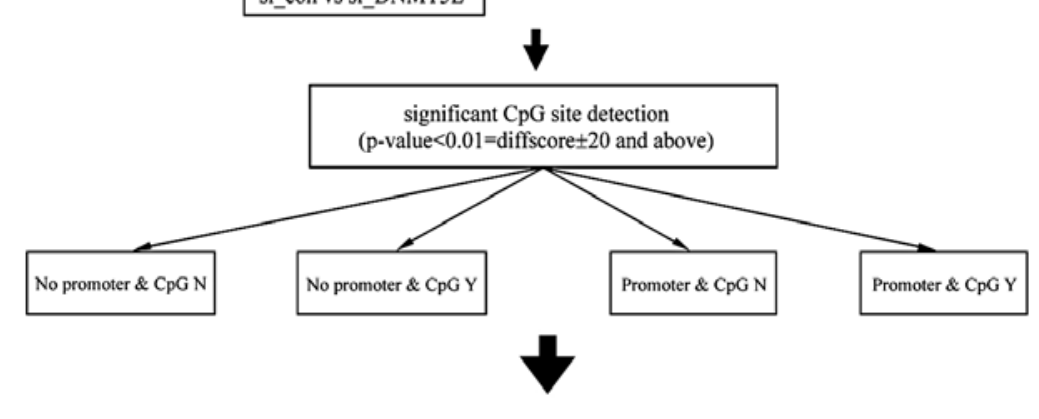

$|\Delta \beta| \geq 0.17$ significant difference of methylation
B

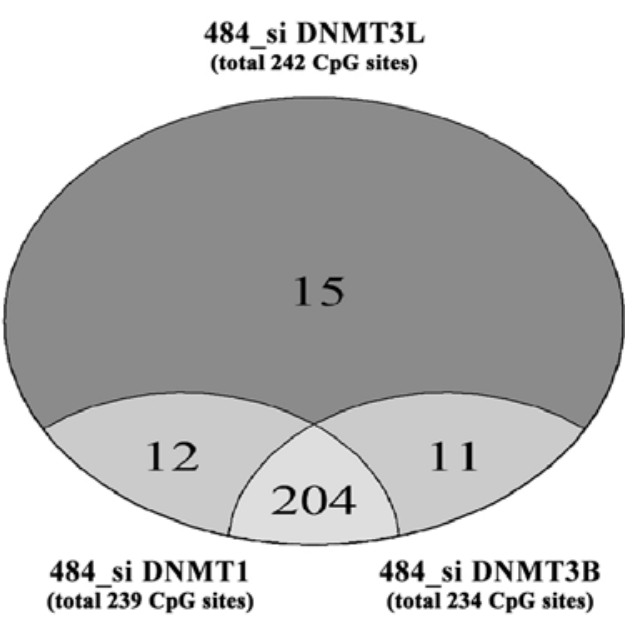

Figure 4. The scheme of analysis for Illumina Goldengate methylation array results and grouping. (A) Targets with detection P>0.05 were excluded among 1,505 targets. A total of 1,502 targets were used in the final analysis. The false discovery rate (FDR) was controlled by adjusting the P-value using the Benjamini-Hochberg algorithm. The classification and grouping of data were conducted on the basis of $>0.17$ differences in methylation level ( $\triangle \Delta ß \mid \geq 0.17$ ), when compared with the controls, and the presence of $\mathrm{CpG}$ islands in the promoter region. (B) With CpG sites evidencing significant alterations in methylation levels, they are grouped by the targets of each DNMT. 
Table III. Fifteen CpG sites detected significant alterations in methylation levels only by siDNMT3L.

\begin{tabular}{|c|c|c|c|c|c|c|}
\hline \multirow[b]{2}{*}{ Target ID } & \multirow[b]{2}{*}{ RefSeq_NM } & \multicolumn{2}{|c|}{ Methylation level } & \multirow[b]{2}{*}{ Delta_beta } & \multirow[b]{2}{*}{ Function } & \multirow{2}{*}{$\begin{array}{l}\mathrm{CpG} \\
\text { island }\end{array}$} \\
\hline & & siCon.AVG_Beta & siDNMT3L.AVG_Beta & & & \\
\hline BAX_E281_R & NM_138765.2 & 0.61 & 0.39 & 0.22 & Apoptosis & Yes \\
\hline EGF_P413_F & NM_001963.2 & 0.92 & 0.73 & 0.19 & Growth factor & No \\
\hline GUCY2F_P255_F & NM_001522.1 & 0.65 & 0.28 & 0.37 & Guanylate cyclase & No \\
\hline HLA-F_E402_F & NM_018950.1 & 0.90 & 0.73 & 0.17 & Immunity & Yes \\
\hline MKRN4_E249_R & & 0.81 & 0.57 & 0.24 & Unkown & Yes \\
\hline PLA2G2A_P528_F & NM_000300.2 & 0.57 & 0.32 & 0.25 & Phospholipase & No \\
\hline SFN_P248_F & NM_006142.3 & 0.75 & 0.53 & 0.22 & Chaperone & No \\
\hline SHB_P473_R & NM_003028.1 & 0.20 & 0.03 & 0.17 & Unkown & Yes \\
\hline TDG_E129_F & NM_001008411.1 & 0.72 & 0.48 & 0.24 & DNA glycosylase & Yes \\
\hline B3GALT5_E246_R & NM_033170.1 & 0.27 & 0.09 & 0.18 & Protein modification & No \\
\hline CDKN1A_P242_F & NM_000389.2 & 0.34 & 0.11 & 0.23 & Kinase inhibitor & Yes \\
\hline DSG1_P159_R & NM_001942.1 & 0.42 & 0.16 & 0.26 & Cell adhesion & No \\
\hline INS_P248_F & NM_000207.1 & 0.84 & 0.66 & 0.18 & Peptide hormone & No \\
\hline NOS2A_P288_R & NM_000625.3 & 0.70 & 0.46 & 0.24 & NO synthase & No \\
\hline PLA2G2A_E268_F & NM_000300.2 & 0.55 & 0.76 & -0.21 & Phospholipase & No \\
\hline
\end{tabular}

Beta-value means methylation levels in CpG sites, ranging from 0 in the case of completely unmethylated sites to 1 in completely methylated sites. Delta_beta means the numerical difference between the $\beta$ value of the sample and of the control.

Considering that the knockdown of DNMT3L also induced a modest reduction of DNMT3B (Fig. 1C), DNMT3B may also contribute to the transcriptional regulation of the genes that were affected by DNMT3L. Therefore, in order to eliminate this possibility and to identify only the target genes of DNMT3L, we selected genes that were significantly altered as the result of DNMT3L suppression but exhibited no changes after suppression of DNMT1 or DNMT3B. As a result of our analysis, we determined that $15 \mathrm{CpG}$ sites (14 genes) evidenced significant alterations in methylation levels following treatment with siDNMT3L alone, thus suggesting that these genes might be regulated principally by DNMT3L (Table III). We also compiled a list of genes that were regulated by the suppression of DNMT3B only or both DNMT3B and DNMT3L, but not DNMT1 (Fig. 4B; Tables IV and $\mathrm{V}$ ).

$T D G$ is regulated primarily by DNMT3L. To validate the results of our methylation microarray trials, we conducted quantitative real-time PCR analysis for some genes that had been randomly selected from each group, as summarized in Tables III-V. In our methylation microarray analyses, NEFL and IPF1 methylations were commonly reduced following treatment with either siDNMT3B or siDNMT3L (Fig. 5A). According to the changes in methylation status, real-time PCR experiments demonstrated that the mRNA expression levels of NEFL and IPF1 were increased in either DNMT3B or DNMT3L knockdown cells (Fig. 5B). However, the methylation levels of BCL2A1 were not reduced, but were rather increased, even though DNMT3B was down-regulated as the result of siRNA treatment (Fig. 5A). Consistent with this result, the expression of BCL2A1 was also reduced in DNMT3B knockdown cells (Fig. 5B).

Among $15 \mathrm{CpG}$ sites (14 genes) regulated by DNMT3L knockdown, we selected TDG for further study. TDG is one of the enzymes involved in the base excision repair of damaged DNA (17). TDG harbored a CpG island within their promoter regions (Fig. 6A), and the methylation levels of it were altered by $>20 \%$ as the result of DNMT3L knockdown in SNU484 cells (Table III). Using methylation microarrays, we determined that the methylation levels of TDG were reduced in DNMT3L knockdown cells as compared to the control cells, by $24 \%$ (Fig. 6B). To further confirm the results of our methylation microarray experiments and to analyze the relationship between the altered methylation levels and gene expression, MSP was conducted for TDG. The map of the $\mathrm{CpG}$ island of TDG and the region for MSP analysis are provided in Fig. 6A. The methylation levels of TDG were reduced gradually in SNU484 cells treated with DNMT3L siRNA, as compared with the control siRNA (Fig. 6C). The mRNA expression levels of TDG were also increased in correlation with the reduction in methylation levels (Fig. 6D). In order to challenge or reinforce these results, we attempted to determine whether the expression of TDG was affected by DNMT3L knockdown in another DNMT3L-expressing gastric cancer cell line, MKN28 cells. As anticipated, the TDG 
Table IV. Eleven CpG sites detected significant difference in methylation levels by only siDNMT3B.

\begin{tabular}{|c|c|c|c|c|c|c|}
\hline \multirow[b]{2}{*}{ Target ID } & \multirow[b]{2}{*}{ RefSeq_NM } & \multicolumn{2}{|c|}{ Methylation level } & \multirow[b]{2}{*}{ Delta_beta } & \multirow[b]{2}{*}{ Function } & \multirow{2}{*}{$\begin{array}{l}\mathrm{CpG} \\
\text { island }\end{array}$} \\
\hline & & siCon.AVG_Beta & siDNMT3B.AVG_Beta & & & \\
\hline ABCA1_E120_R & NM_005502.2 & 0.88 & 0.65 & 0.23 & Transporter & Yes \\
\hline BCL2A1_P1127_R & NM_004049.2 & 0.79 & 0.97 & -0.18 & Anti-apoptosis & Yes \\
\hline CDH17_E31_F & NM_004063.2 & 0.66 & 0.49 & 0.17 & Cell adhesion & No \\
\hline CLDN4_P1120_R & NM_001305.3 & 0.69 & 0.87 & -0.18 & Cell structure, mobility & No \\
\hline EFNB3_E17_R & NM_001406.3 & 0.97 & 0.76 & 0.21 & Signaling molecule & Yes \\
\hline GPR116_E328_R & NM_015234.3 & 0.74 & 0.51 & 0.23 & Receptor & No \\
\hline HOXA9_P303_F & NM_002142.3 & 0.44 & 0.25 & 0.19 & Transcriptional factor activity & Yes \\
\hline IRAK3_E130_F & NM_007199.1 & 0.83 & 0.61 & 0.22 & Receptor & Yes \\
\hline MCF2_E195_F & NM_005369.2 & 0.41 & 0.17 & 0.24 & Signaling molecule & No \\
\hline NEU1_P745_F & NM_000434.2 & 0.54 & 0.25 & 0.29 & Hydrolase, metabolism & Yes \\
\hline RET_P717_F & NM_020630.3 & 0.40 & 0.09 & 0.31 & Receptor & No \\
\hline
\end{tabular}

A

IPF1

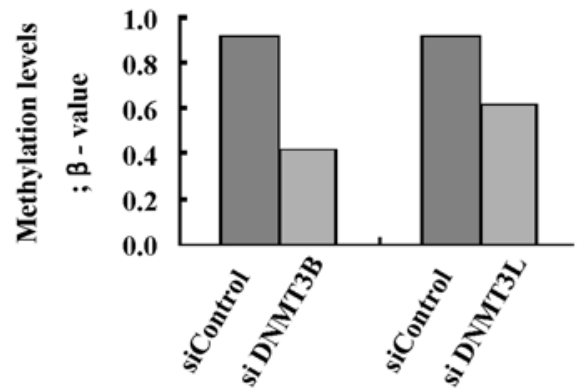

B

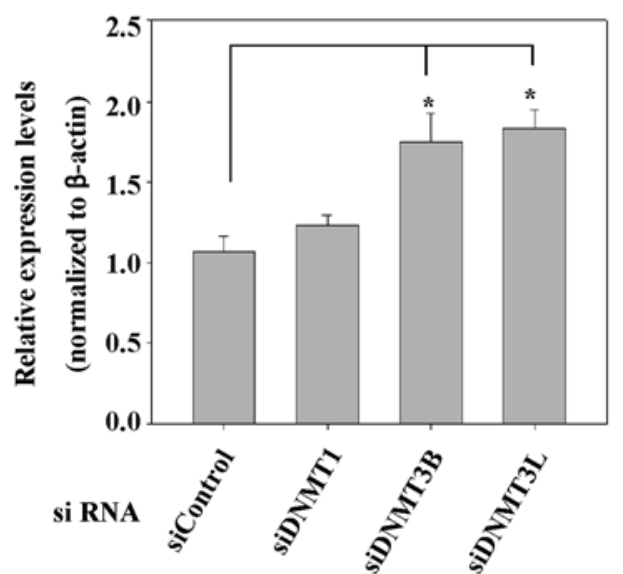

NEFL

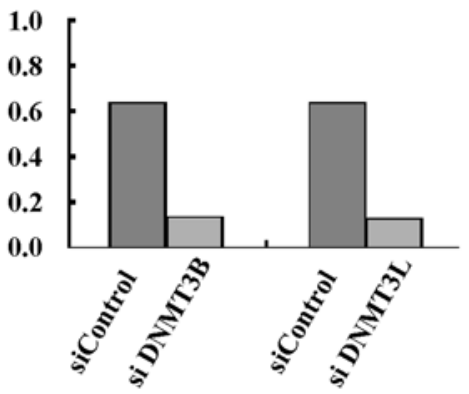

NEFL

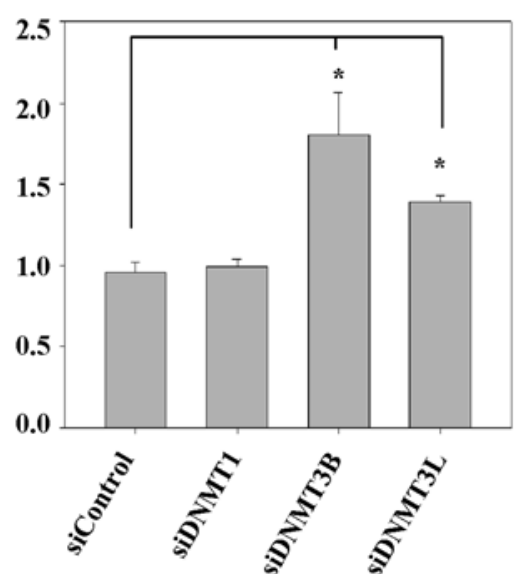

BCL2A1

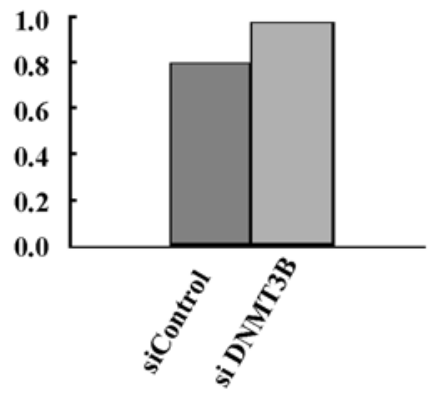

BCL2A1

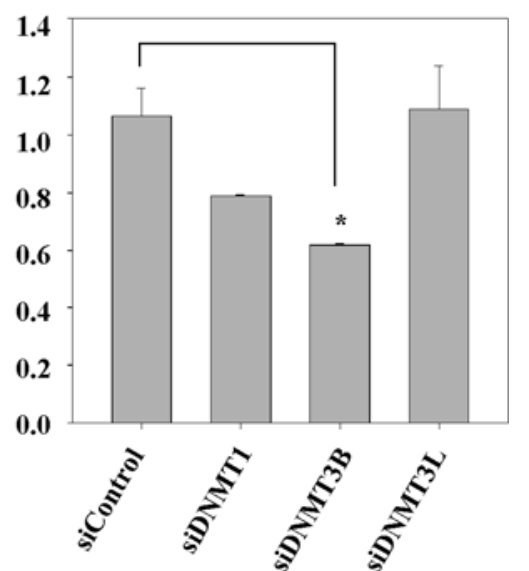

Figure 5. Correlation between methylation microarray results and mRNA levels. (A) The methylation level of each gene was determined using the methylation microarray. B-values show the methylation levels in CpG sites. (B) Real-time RT-PCR was conducted using specific primers for each gene. The bars represent the means $\pm \mathrm{SD}$ and control siRNA-treated samples are set to a value of $1.0 ;{ }^{*} \mathrm{P}<0.05$.

transcripts were up-regulated by DNMT3L knockdown in the MKN28 cells (Fig. 6D).

The loss of DNA methylation at the DNMT3L promoter in cervical cancer was recently observed (10), and its expression was associated with epigenetic modification (18). We also determined that certain gastric cancer cell lines did not express DNMT3L and that 5-aza-dC treatment re-induced DNMT3L expression (Fig. 7A). However, the expression of 
Table V. CpG sites detected significant differences in methylation levels by only both siDNMT3B and siDNMT3L.

\begin{tabular}{|c|c|c|c|c|c|c|}
\hline \multirow[b]{2}{*}{ Target ID } & \multirow[b]{2}{*}{ RefSeq_NM } & \multicolumn{2}{|c|}{ Methylation level } & \multirow[b]{2}{*}{ Delta_beta } & \multirow[b]{2}{*}{ Function } & \multirow{2}{*}{$\begin{array}{l}\mathrm{CpG} \\
\text { island }\end{array}$} \\
\hline & & siCon.AVG_Beta & $\begin{array}{l}\text { siDNMT3L.AVG_Beta } \\
\text { (siDNMT3B.AVG_Beta) }\end{array}$ & & & \\
\hline DNMT3B_P352_R & NM_175848.1 & 0.79 & $0.53(0.58)$ & $0.26(0.21)$ & DNA methyltransferase & No \\
\hline IGFBP5_E144_F & NM_000599.2 & 0.32 & $0.03(0.02)$ & $0.29(0.30)$ & Growth factor homeostasis & Yes \\
\hline IPF1_P234_F & NM_000209.1 & 0.91 & $0.61(0.41)$ & $0.30(0.49)$ & Transcription factor & Yes \\
\hline ITK_E166_R & NM_005546.3 & 0.56 & $0.02(0.02)$ & $0.54(0.54)$ & Protein kinase; Apoptosis & No \\
\hline NEFL_E23_R & NM_006158.1 & 0.63 & $0.12(0.13)$ & $0.51(0.50)$ & cytoskeletal protein & Yes \\
\hline PDGFA_P841_R & XM_926001.1 & 0.22 & $0.03(0.03)$ & $0.19(0.19)$ & Growh factor activity & Yes \\
\hline PTHR1_P258_F & NM_000316.2 & 0.36 & $0.64(0.59)$ & $-0.28(-0.23)$ & Receptor & No \\
\hline RIPK4_E166_F & NM_020639.2 & 0.43 & $0.21(0.23)$ & $0.22(0.20)$ & Protein kinase; Apoptosis & Yes \\
\hline TCF4_P317_F & NM_003199.1 & 0.71 & $0.53(0.24)$ & $0.18(0.47)$ & Transcription factor & Yes \\
\hline TNC_P198_F & NM_002160.1 & 0.84 & $0(0)$ & $0.84(0.84)$ & Extracellular matrix & Yes \\
\hline TSG101_P257_R & NM_006292.2 & 0.74 & $0.49(0.01)$ & $0.24(0.72)$ & Ubiquitin-protein ligase & Yes \\
\hline
\end{tabular}

A

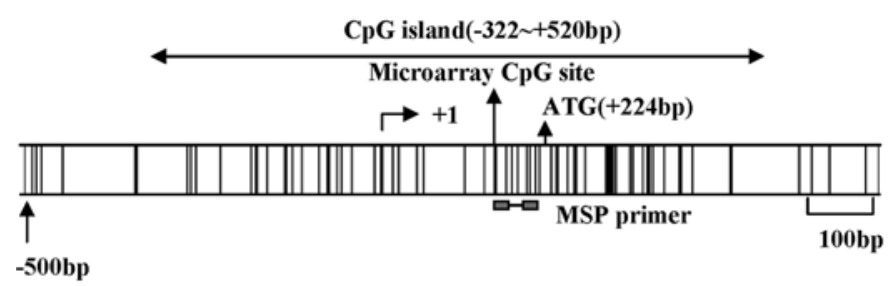

B

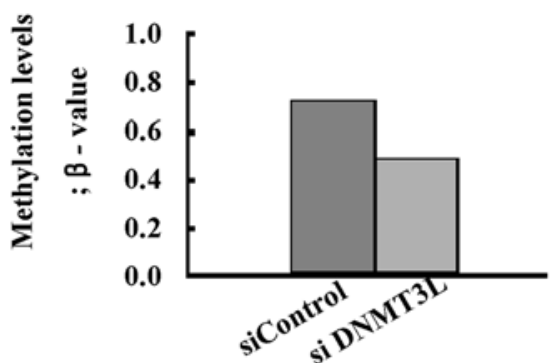

C

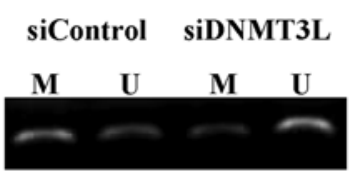

$\mathrm{D}$
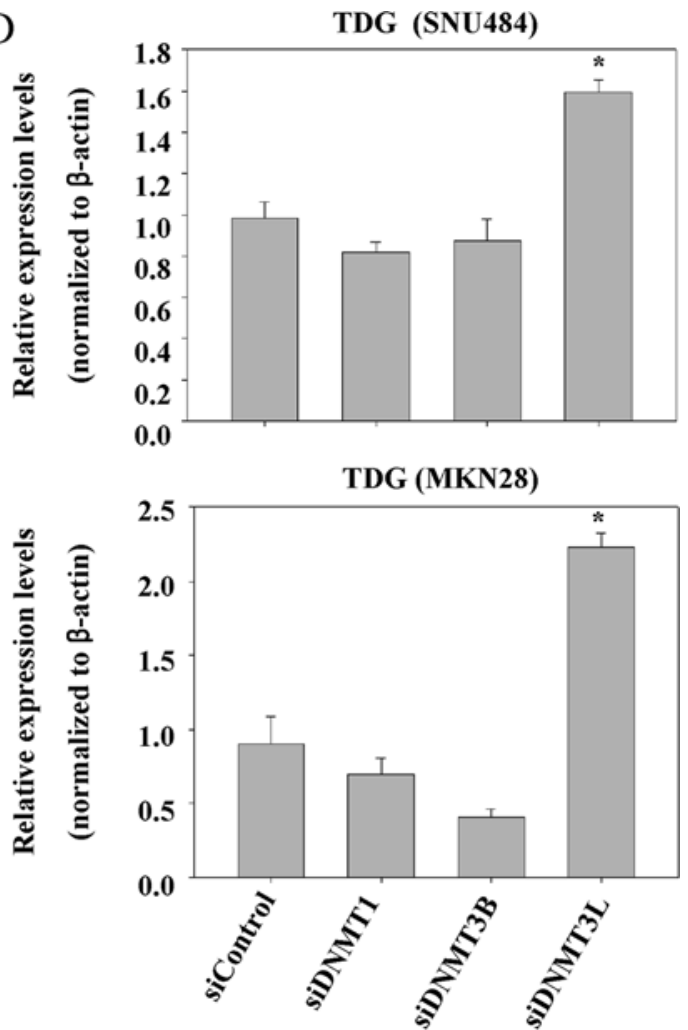

Figure 6. TDG transcripts are upregulated by DNMT3L knockdown. (A) A map of the CpG island of the TDG gene. Vertical bars mean the locations of the CpG sites. The regions of methylation microarray and MSP are shown. (B) Methylation levels of TDG from methylation microarray results. (C) Methylation status of the TDG in DNMT3L knockdown SNU484 cells. The methylation level of TDG was determined using methylation-specific PCR. (D) mRNA levels of TDG were determined using real-time RT-PCR; ${ }^{*} \mathrm{P}<0.05$.

DNMT1 and DNMT3B were down-regulated as the result of 5 -aza-dC treatment $(19,20)$, thus indicating that 5 -aza-dC exerts an opposite effect on DNMT1/3B and DNMT3L. Since TDG was up-regulated only in the DNMT3L knockdown cell lines, and not the DNMT1 or DNMT3B knockdown lines (Fig. 6D), we further attempted to ascertain whether 5 -aza-dC treatment resulted in decreased mRNA levels of TDG as a result of the increase in DNMT3L expression in 
A
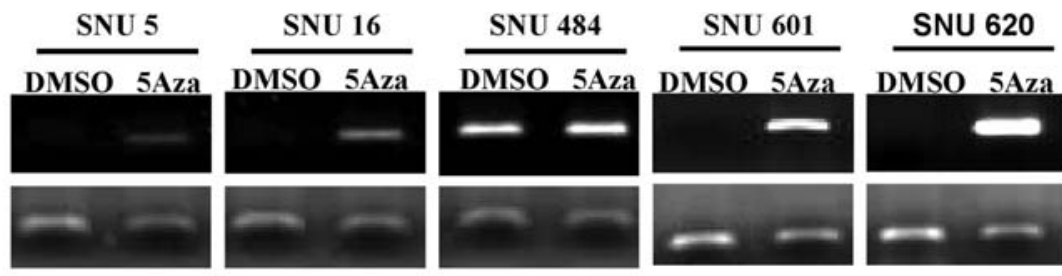

DNMT3L

B

C

\section{GAPDH}

TDG
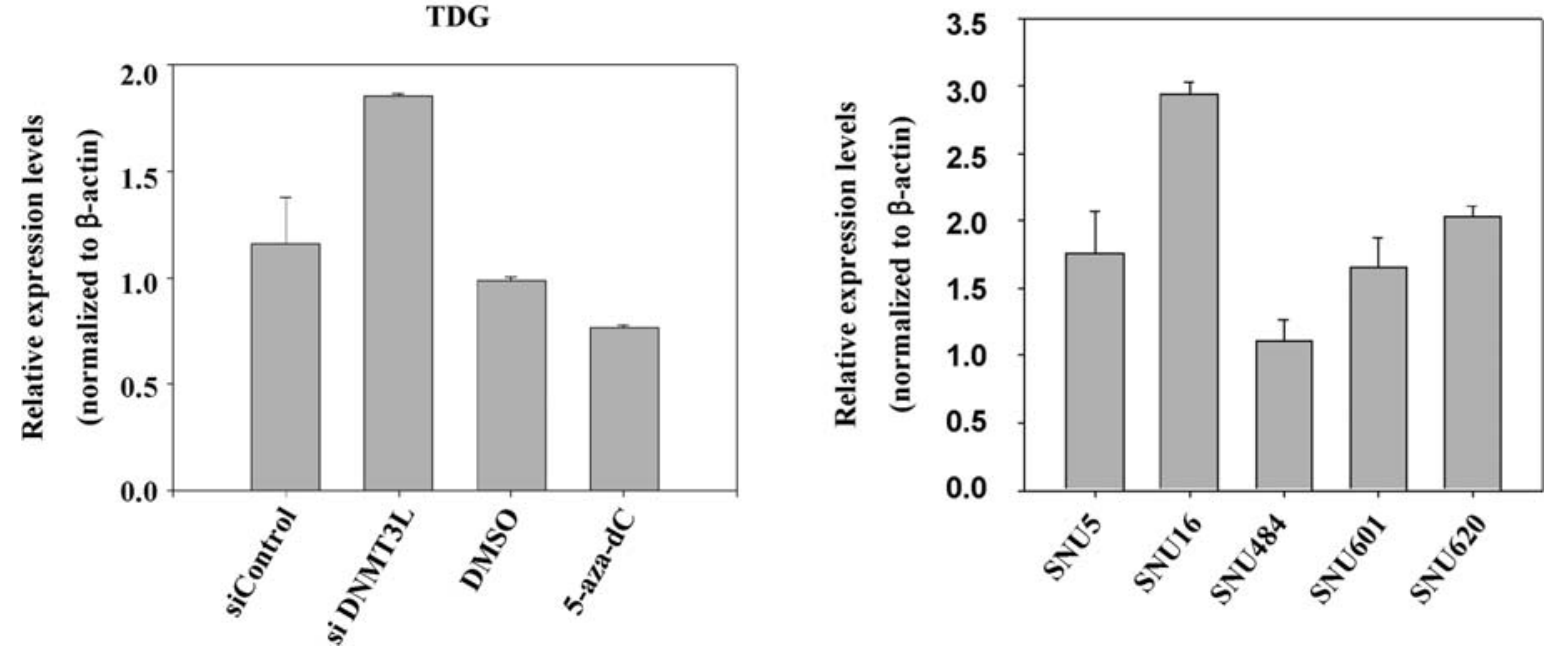

Figure 7. DNMT3L contributes primarily to TDG regulation in cancer cells. (A) SNU gastric cancer cells were treated every $48 \mathrm{~h}$ for 4 days with DMSO or $5 \mu \mathrm{M}$ of 5-aza-dC. DNMT3L mRNA levels were detected via RT-PCR. GAPDH served as a loading control. (B) Relative mRNA expression levels of TDG in SNU484 cells treated with indicated reagents were determined via real-time RT-PCR. Expression levels are shown as relative values to the level in each of the control siRNA and DMSO, which were set to 1 .

SNU484 cells. TDG mRNA levels were reduced in the cells treated with 5-aza-dC versus DMSO (Fig. 7B). On the basis of these results, we subsequently attempted to determine whether the TDG basal expression levels in gastric cancer cell lines not expressing DNMT3L were higher than in the SNU484 cells expressing DNMT3L. As had been expected, the TDG mRNA levels in most gastric cancer cell lines were higher than in the SNU484 cells (Fig. 7C). These results show that DNMT3L may perform an important role in the regulation of TDG in cancer cells.

\section{Discussion}

The results of this study indicate that DNMT3L, which was previously considered a minor enzyme, may actually be quite an important enzyme in the context of cancer development. Most notably, a recent study demonstrated that DNMT3L was also overexpressed as a result of its promoter hypomethylation in cervical cancer, thus suggesting that DNMT3L may function as a putative oncogene during cancer development (10). Another previous study demonstrated that DNMT3L was down-regulated in accordance with the increase in its promoter methylation during the in vitro differentiation of ES cells, and that the abnormal regulation of DNMT3L could disrupt normal development (12). Collectively, our results indicate that DNMT3L expression in cancer cells may contribute to alterations in the DNA methylation and expression of certain genes.

Therefore, our study focused principally on the genes affected by DNMT3L, primarily to expand our current knowledge regarding the role of DNMT3L during tumorigenesis. We were surprised to find that DNMT3L siRNA increased apoptosis in SNU484 cells to the same degree as DNMT1 siRNA, but DNMT3B siRNA did not alter the incidence of apoptosis, as compared to control siRNA (Fig. 3). Considering that DNMT1 is a major enzyme in the maintenance of aberrant methylation in cancer (3) and has characteristics that distinguish it from the DNMT3A and DNMT3B de novo methyltransferases (4), this result implies that DNMT3L may be involved in tumor proliferation.

The methylation microarray data were grouped in accordance with the $\mathrm{CpG}$ sites affected by each DNMT. Since DNMT3L was traditionally considered a minor enzyme, in comparison to DNMT1 and DNMT3B, we were surprised to observe that the methylation of $242 \mathrm{CpG}$ sites was significantly altered by DNMT3L knockdown. However, it does make some sense that many genes were affected by DNMT3L, as DNMT3L is a regulatory enzyme that stimulates the activity of other DNMTs (6), and the DNMTs are all somewhat intertwined with one another, as was confirmed by our results (Fig. 1C). This also demonstrates why 227 of 242 CpG sites overlapped with other groups affected by DNMT1 or DNMT3B (Fig. 4B).

To understand the role of DNMT3L, it is also worth including genes affected by the knockdown of DNMT3B, as DNMT3L is related more closely to DNMT3B than to DNMT1 in terms of their interaction and the role of de novo methylation (21). Furthermore, our results showed that DNMT3L mRNA levels were positively correlated with the levels of DNMT3B mRNA (Fig. 1C). Therefore, we randomly 
selected one gene (BCL2A) from 6 CpG sites, all of which were affected only by DNMT3B knockdown and harbored a promoter-region $\mathrm{CpG}$ island (Table IV), as well as two genes (NEFL and IPF1) from $8 \mathrm{CpG}$ sites, all of which were affected by DNMT3L or DNMT3B knockdown and also harbored a $\mathrm{CpG}$ island within the promoter region (Table V).

In the case of BCL2A, the methylation levels of its target $\mathrm{CpG}$ sites were increased, despite the down-regulation of DNMT3B. The levels of BCL2A mRNA expression were also reduced, which correlated with observed increases in its methylation levels. Our results may validate, to some degree, a recent study showing that certain genes are down-regulated after exposure to the demethylating agent, 5-aza-dC (22). The anti-apoptotic BCL2 family, which includes BCL2A, is commonly overexpressed in a variety of cancers and members of this family function as oncoproteins that inhibit apoptosis until the cells can accrue mutations sufficient for further tumorigenesis (23). Therefore, BCL2 family members are considered molecules that can be targeted in order to suppress tumor development (23). In our study, we demonstrated that BCL2A was down-regulated only in the DNMT3B knockdown cell lines (Fig. 5B), thus suggesting that the overexpression of DNMT3B in cancer may contribute to the inhibition of apoptosis and the proliferation of cancer cells.

DNMT3L expression in SNU484 gastric cancer cells is a matter of some significance, in that SNU484 cells are primary cancer cells, which can undergo nuclear reprogramming as the result of the overexpression of DNMT3L, which is involved in cancer progression (11). According to the epigenetic progenitor model, tumor heterogeneity, which includes metastatic capability and drug resistance, is an intrinsic and plastic property of primary tumors arising as the result of epigenetic change (24). Therefore, because the overexpression and inappropriate expression of DNMT3L might result in aberrant epigenetic gene activation and silencing, DNMT3L also may be a tumor progenitor gene, such as OCT4 and Nanog $(24,25)$.

Most of all, among $15 \mathrm{CpG}$ sites affected by only DNMT3L, we narrowed our focus down to $5 \mathrm{CpG}$ sites, which were located in a $\mathrm{CpG}$ island of promoter region and have well-characterized functions. Among these $5 \mathrm{CpG}$ sites, we confirmed that TDG was overexpressed only in the DNMT3L knockdown cells. A recent study of TDG indicated that DNMT3A and DNMT3B appear to stimulate glycosylase activity via interaction with TDG, whereas TDG inhibits the methylation activity of DNMT3A in vitro (26). In addition, the efficiency of TDG was reduced in the absence of DNMTs (27). However, our results showed that TDG appears to be regulated negatively by DNMT3L, which is supported by the reduction in methylation levels following DNMT3L knockdown in the methylation microarray and MSP (Fig. 6B and $\mathrm{C}$ ). One possible explanation is that the down-regulation of DNMT3L owing to the overexpression of DNMT3A and DNMT3B might result in the up-regulation of stimulation of TDG, since the promoter methylation and silencing of DNMT3L are mediated by DNMT3A and DNMT3B (12). This conclusion might also be supported by the fact that, whereas DNMT3L was down-regulated owing to high levels of DNMT3B in the epiblasts from E5.5 to E7.5 (12), TDG was expressed from 7.5-13.5 days post-coitum (d.p.c) (28).
Some research groups have demonstrated that expression of DNMT1, DNMT3A and DNMT3B were reduced after treatment with 5 -aza-dC $(19,20)$. In the case of DNMT3L, however, silenced-DNMT3L was re-induced by 5-aza-dC treatment (Fig. 7A). Since TDG was up-regulated by DNMT3L knockdown, the induction of DNMT3L by 5-aza-dC treatment could not be dismissed. TDG is responsible for a specific base-excision repair pathway that corrects $\mathrm{G} / \mathrm{T}$ mismatches arising as the result of methylcytosine deamination, thereby suggesting that this epigenetic stability function might contribute to tumor suppression (17). However, the hypermethylation of TDG results in genomic instability and chromosomal aberration, which may contribute to malignancy (29). Thus, 5-aza-dC treatments against cancer may rather promote tumorigenesis by inhibiting repair enzymes that play a significant role in cancer protection. The aforementioned relationship of DNMT3L with DNMT3A/B, TDG, and 5-aza$\mathrm{dC}$ may provide an explanation as to why DNMT3A/B double-null ES cells were highly resistant to 5-aza-dC (19). Therefore, our results reinforce the need for targeted therapies such as treatments with specific DNMT3L inhibitors, which are expected both to minimize the side effects of anti-cancer drugs and to help in achieving outstanding cancer therapy results.

\section{Acknowledgements}

This work was supported by a grant from the National R\&D Program for Cancer Control, Ministry of Health, Welfare and Family Affairs, Republic of Korea (0720540) and the BK21 project from the Ministry of Education and Human Resources Development.

\section{References}

1. Jones PA and Baylin SB: The epigenomics of cancer. Cell 128: 683-692, 2007.

2. Esteller M: Cancer epigenomics: DNA methylomes and histonemodification maps. Nat Rev Genet 8: 286-298, 2007.

3. Robert MF, Morin S, Beaulieu N, et al: DNMT1 is required to maintain CpG methylation and aberrant gene silencing in human cancer cells. Nat Genet 33: 61-65, 2003.

4. Okano M, Bell DW, Haber DA and Li E: DNA methyltransferases Dnmt3a and Dnmt3b are essential for de novo methylation and mammalian development. Cell 99: 247-257, 1999.

5. Rhee I, Bachman KE, Park BH, et al: DNMT1 and DNMT3b cooperate to silence genes in human cancer cells. Nature 416: 552-556, 2002.

6. Suetake I, Shinozaki F, Miyagawa J, Takeshima H and Tajima S: DNMT3L stimulates the DNA methylation activity of Dnmt3a and Dnmt3b through a direct interaction. J Biol Chem 279: 27816-27823, 2004.

7. Gowher H, Liebert K, Hermann A, Xu G and Jeltsch A: Mechanism of stimulation of catalytic activity of Dnmt3A and Dnmt3B DNA-(cytosine-C5)-methyltransferases by Dnmt3L. J Biol Chem 280: 13341-13348, 2005.

8. Bourc'his D and Bestor TH: Meiotic catastrophe and retrotransposon reactivation in male germ cells lacking Dnmt3L. Nature 431: 96-99, 2004.

9. Webster KE, O'Bryan MK, Fletcher S, et al: Meiotic and epigenetic defects in Dnmt3L-knockout mouse spermatogenesis. Proc Natl Acad Sci USA 102: 4068-4073, 2005.

10. Gokul G, Gautami B, Malathi S, et al: DNA methylation profile at the DNMT3L promoter: a potential biomarker for cervical cancer. Epigenetics 2: 80-85, 2007.

11. Gokul G, Ramakrishna G and Khosla S: Reprogramming of HeLa cells upon DNMT3L overexpression mimics carcinogenesis. Epigenetics 4: 322-329, 2009. 
12. Hu YG, Hirasawa R, Hu JL, et al: Regulation of DNA methylation activity through Dnmt3L promoter methylation by Dnmt3 enzymes in embryonic development. Hum Mol Genet 17: 2654-2664, 2008.

13. Bibikova M, Lin Z, Zhou L, et al: High-throughput DNA methylation profiling using universal bead arrays. Genome Res 16: 383-393, 2006.

14. Robertson KD, Uzvolgyi E, Liang G, et al: The human DNA methyltransferases (DNMTs) 1, 3a and 3b: coordinate mRNA expression in normal tissues and overexpression in tumors. Nucleic Acids Res 27: 2291-2298, 1999

15. Herman JG, Graff JR, Myohanen S, Nelkin BD and Baylin SB: Methylation-specific PCR: a novel PCR assay for methylation status of CpG islands. Proc Natl Acad Sci USA 93: 9821-9826, 1996.

16. Nimura $\mathrm{K}$, Ishida $\mathrm{C}$, Koriyama $\mathrm{H}$, et al: Dnmt3a2 targets endogenous Dnmt3L to ES cell chromatin and induces regional DNA methylation. Genes Cells 11: 1225-1237, 2006.

17. Cortazar D, Kunz C, Saito Y, Steinacher R and Schar P: The enigmatic thymine DNA glycosylase. DNA Repair (Amst) 6: 489-504, 2007.

18. Aapola U, Maenpaa K, Kaipia A and Peterson P: Epigenetic modifications affect Dnmt3L expression. Biochem J 380 705-713, 2004

19. Oka M, Meacham AM, Hamazaki T, Rodic N, Chang LJ and Terada N: De novo DNA methyltransferases Dnmt3a and Dnmt3b primarily mediate the cytotoxic effect of 5-aza-2'deoxycytidine. Oncogene 24: 3091-3099, 2005.

20. Ghoshal K, Datta J, Majumder S, et al: 5-Aza-deoxycytidine induces selective degradation of DNA methyltransferase 1 by a proteasomal pathway that requires the KEN box, bromoadjacent homology domain, and nuclear localization signal. Mol Cell Biol 25: 4727-4741, 2005.
21. Chen ZX, Mann JR, Hsieh CL, Riggs AD and Chedin F: Physical and functional interactions between the human DNMT3L protein and members of the de novo methyltransferase family. J Cell Biochem 95: 902-917, 2005.

22. Gius D, Cui H, Bradbury CM, et al: Distinct effects on gene expression of chemical and genetic manipulation of the cancer epigenome revealed by a multimodality approach. Cancer Cell 6: 361-371, 2004.

23. Oltersdorf T, Elmore SW, Shoemaker AR, et al: An inhibitor of Bcl-2 family proteins induces regression of solid tumours. Nature 435: 677-681, 2005.

24. Feinberg AP, Ohlsson R and Henikoff S: The epigenetic progenitor origin of human cancer. Nat Rev Genet 7: 21-33, 2006.

25. Ezeh UI, Turek PJ, Reijo RA and Clark AT: Human embryonic stem cell genes OCT4, NANOG, STELLAR, and GDF3 are expressed in both seminoma and breast carcinoma. Cancer 104: 2255-2265, 2005

26. Li YQ, Zhou PZ, Zheng XD, Walsh CP and Xu GL: Association of Dnmt3a and thymine DNA glycosylase links DNA methylation with base-excision repair. Nucleic Acids Res 35: 390-400, 2007.

27. Boland MJ and Christman JK: Characterization of Dnmt3b: thymine-DNA glycosylase interaction and stimulation of thymine glycosylase-mediated repair by DNA methyltransferase(s) and RNA. J Mol Biol 379: 492-504, 2008.

28. Niederreither K, Harbers M, Chambon P and Dolle P: Expression of T:G mismatch-specific thymidine-DNA glycosylase and DNA methyl transferase genes during development and tumorigenesis. Oncogene 17: 1577-1585, 1998.

29. Peng B, Hurt EM, Hodge DR, Thomas SB and Farrar WL: DNA hypermethylation and partial gene silencing of human thymine-DNA glycosylase in multiple myeloma cell lines. Epigenetics 1: 138-145, 2006. 